\title{
Las organizaciones étnicas y el discurso de la identidad en el norte de Chile, 1980-2000
}

Hans Gundermann K. ${ }^{2}$

\section{RESUMEN}

Se analiza el surgimiento entre 1980-2000 de la etnicidad como dimensión otorgadora de sentido y perspectiva a actores sociales andinos en el norte de Chile. Se comenta, asimismo, la constitución de esos actores con arreglo a circunstancias políticas y a los discursos de la identidad. La relación de este proceso con la acción de los gobiernos de la Concertación es también considerada. La diferenciación de funciones burocráticas y de representación hacia finales del período representa otro hecho caracterizador de la evolución de esos actores. Un corolario de todo lo anterior es el bloqueo de lo que se inició como un movimiento social incipiente.

\begin{abstract}
In this paper, the author analyses the emergency of ethnicity between the years 1980 and 2000, as a dimension providing a sense and perspective to the andean social actors in northern Chile. The formation of such actors is also dealt with, in connection with the political circumstances and identity discourse. The relationship between this process and the Concertación (the coalition now in power's administrations is also considered). The differences of bureaucratic and representation functions toward the end of this period is another fact that characterizes the evolution of the actors mentioned above. A corollary of all is the blockade of what began as an incipient social movement.
\end{abstract}

\section{Introducción}

Este trabajo trata de la etnogénesis de los pueblos andinos del norte de Chile, de su conformación como actores étnicos durante las dos últimas décadas ${ }^{3}$. Ese proceso tiene como protagonistas principales a un grupo de organizaciones andinas (aymaras, atacameñas y collas) y al Estado chileno en las postrimerías del régimen militar y en los gobiernos de transición hacia una democracia más plena. De esta manera, el Estado es un agente siempre presente en el proceso, cumpliendo un papel de negación, primero, y de promoción de la etnicidad, después. El desarrollo de la exposición considera tres capítulos. En el primero se trata de la innovación socio política que representa en la región norte del país la etnogénesis andina. En el segundo se analiza el pacto logrado por la Concertación de Partidos por la Democracia, coalición gobernante desde hace una década y que sucede a la dictadura de Pinochet, y los pueblos indígenas del país, entre ellos las etnias 
andinas. En la tercera, finalmente, se aborda la separación de funciones burocráticas y de representación que un mismo grupo de intelectuales y dirigentes andinos venía efectuando, dando origen además a una representación más diferenciada. Se analiza críticamente todo este proceso destacando la situación de bloqueo en que se encuentra aquello que a finales de la década de 1980 constituyó un movimiento social en ciernes.

\section{La etnogénesis andina, innovación y doble para- doja}

La constitución de aymaras, ${ }^{4}$ atacameños ${ }^{5}$ y collas ${ }^{6}$ como actores étnicos provistos de una conciencia de identidad étnica y de demandas que interpelan a la sociedad y al Estado, representa tanto una novedad histórica como una paradoja. En efecto, durante los 120 años de anexión de los territorios de Arica y Tarapacá (antes pertenecientes al Perú) y de Antofagasta (perteneciente a Bolivia hasta 1879) la población andina chilena, genéricamente hablando, ${ }^{7}$ nunca se planteó previamente (al menos de manera directa) ante el Estado y la restante sociedad como pueblos y, sobre todo, con una interpelación y una demanda como tales. Por otra parte, la acción colectiva andina durante las décadas anteriores a los años de la etnogénesis se orientó en un sentido precisamente opuesto al de esa autoafirmación y ac-

4 La población aymara chilena asciende a unas 50 mil personas aproximadamente, radicadas en su gran mayoría en la Región de Tarapacá, extremo norte de Chile, representando alrededor de un $12 \%$ de la población regional. Activas migraciones que tuvieron lugar durante la segunda mitad del S.XX modificaron la distribución rural del asentamiento. Actualmente, los aymaras son urbanos en una proporción global de $3: 1$. Su conformación como minoría étnica nacional arranca de la Guerra del Pacífico, la cual desde 1879 deja a Tarapacá en poder de Chile, segmentando la población indígena del sur del Perú en dos grupos, el más meridional de los cuales es el que queda sujeto a la soberanía ejercida por el Estado chileno.

5 Los atacameños ascienden a unas 10 mil personas (las cifras son sólo aproximadas por falta de censos) radicadas en comunidades campesinas del alto río Loa y de la cuenca del salar de Atacama en la zona interior de la Región de Antofagasta. Como en el caso aymara, la mayoría de la población radica en medios urbanos (Calama, en primer lugar, y en el puerto de Antofagasta, luego). Se conforman como minoría étnica indígena nacional desde la Guerra del Pacífico cuando los territorios ancestrales de esta etnia andina particular pasan junto con sus pobladores al dominio chileno.

6 Se trata de unos cientos de individuos radicados en u oriundos de la cordillera andina en los altos de la provincia de Chañaral y de Copiapó, ahora residentes en su mayoría en centros urbanos como El Salvador y Copiapó. Son los descendientes de familias de pastores migrantes desde el noroeste argentino hace aproximadamente un siglo y que adquieren notoriedad a ción regida según criterios de identidad étnica. Abundando más, señalemos que la paradoja es doble pues, precisamente, en el tránsito por la ruta de la asimilación cultural se crean las condiciones que, en cierto momento y coyuntura, posibilitaron la emergencia de lo étnico como un principio de estructuración de la acción colectiva.

La acción colectiva anterior a la segunda mitad de la década de 1980 tuvo un alcance exclusivamente local y, en menor medida, sectorial. Local, porque se organizó siguiendo los contornos y las posibilidades de la, quizá, principal institución social hasta el presente: la comunidad andina ${ }^{8} \mathrm{y}$ sectorial, por lo menos desde la década de 1960 , con la creación estimulada desde el Estado de organizaciones de productores (cooperativas, comités, asociaciones). Un segmento de la población aymara, ${ }^{9}$ atacameña y colla ${ }^{10}$ eran considerada y se percibía a sí misma de

principio de la década de 1990 luego de su reconocimiento por la entonces Comisión Especial de los Pueblos Indígenas (CEPI).

7 Por tratarse las tres etnias mencionadas de grupos sociales con una fíliación cultural relacionada con el espacio civilizatorio andino.

8 Lejos estamos de asumir una visión tradicionalista de la comunidad. La comunidad andina es históricamente incomprensible sin atender a la creación de una sociedad colonial estamentaria y patrimonialista a partir de mediados del S.XVI, un aspecto de lo cual consistió en la reorganización de la población nativa en reducciones de indios. El marco institucional de esta entidad es castellano, en tanto que numerosos contenidos provienen de la tradición andina precolombina y otros muchos se elaboran en la propia dinámica de conformación de esas unidades locales. Ellas dan origen a las comunidades de indios y en ellas se gesta y reproduce socialmente una matriz cultural. A esa matriz se acude en el presente para representar la tradición andina, sobre la base del reconocimiento que ella ha sufrido transformaciones importantes indeseables, especialmente durante la segunda mitad del S. $\mathrm{XX}$, y de una toma de posición "tradicionalista" o de recuperación de la tradición.

9 Aquella radicada en la alta cordillera y la altiplanicie andina, dedicada principalmente a la ganadería extensiva de ovinos y camélidos sudamericanos (llamas y alpacas).

10 Nos referimos a los pastores de las alturas andinas de la región atacameña y de los altos de Chañaral y Copiapó, quienes eran percibidos por la población atacameña y por la población minera y campesina de las dos últimas zonas genéricamente como "indios" o "collas". En esta zona meridional andina Ios "collas" o "indios collas" eran percibidos como una población advenediza en la vertiente occidental de Ios Andes, proveniente del noroeste argentino, aun cuando objetivamente los atacameños mantuvieron activas relaciones y vínculos con las zonas de origen de estos pastores. La definición de fronteras étnicas sería un proceso tardío, ligado a la migración de pastores de la puna argentina hacia Chile a principios del S.XX, movimiento originado en las presiones para el pago de rentas por uso de pastizales rústicos en sus zonas de origen y, luego de llegados a los altos de Atacama, su subordinación a grupos de poder local de las comunidades atacameñas. 
manera contradictoria como "india". ${ }^{11}$ Esta conciencia los hacía partícipes de una categoría de población percibida externa e internamente según atributos negativos ("atrasado", "incivilizado", "motoso", ${ }^{2}$ marginal, sin educación, etc.). La acción planteada desde esa forma de conciencia, convergente con la de campesinos y pobladores rurales ${ }^{13}$ tenía por objetivos más generales los del "desarrollo" para salir del atraso, los de la "civilización", los de la educación castellanizante, los de la integración territorial, los de la incorporación y la movilidad social, y los del acceso a estándares de vida cuyo modelo era urbano y centralista. De esta manera, siguiendo un marco local y sectorial de acción colectiva, ${ }^{14}$ la población andina no carecía del todo de una referencia de adscripción más general de carácter étnico. Pero, era problemática en el sentido mencionado $\mathrm{y}$, por tanto, no podía constituirse en una pertenencia que informara un eventual curso de acción planteado sólo local y sectorialmente. Deberá esperarse otros tiempos para que surjan líderes y se formen organizaciones que elaboren un discurso y demanda étnica de otro cuño.

La demanda es una de desarrollo en lo económico, de integración y movilidad en lo social, de asimilación en lo cultural y de ciudadanía en lo político. Ella se organiza y procesa sólo local y sectorialmente. Se sostiene en un diagnóstico de

11 La identidad colectiva estructurada en torno a la denominación de "indio" presenta durante momentos republicanos una evidente disociación entre identificación y reconocimiento. Nadie se identifica positivamente como "indio". Hasta muy recientemente, sólo lo hacen negativamente sobre sí mismos un segmento de aymaras de la alta cordillera sujetos a la condición de pastores, al igual que los collas del espacio atacameño. Sobre estos segmento se impone su reconocimiento desde posiciones relativas de poder, pero como un estigma, en primer lugar por la población agricultora aymara y atacameña sobre la población de pastores y, durante el S.XIX y principios del XX, también sobre todos aquellos campesinos agricultores reconocibles como indígenas según marcas de reconocimiento étnico. En segundo lugar, por la población regional se produce un similar reconocimiento, pero ahora ampliando la cobertura de la apelación estigmatizante para incluir también a toda la población andina de los sectores de precordillera y oasis andinos.

12 Que habla imperfectamente la lengua castellana.

13 Común esta última a toda la población andina; es decir, tanto aquella que se consideraba "india" como la que se ponía un paso más allá de ella, bajo el paraguas general de "chileno".

14 Nos referimos, en un caso, a la gestión ante el Estado y a la autogestión por escuelas, caminos, créditos, salud, subsidios y créditos agrícolas, etc. En el segundo, a la demanda de asistencia técnica, tecnología, crédito, mercados, tierra y aguas que es característica de los pequeños productores agropecuarios. Ambos tipos de demandas son específicas y siguen dinámicas propias, aunque se traslapan en algunos aspectos puntuales. atraso como falta de desarrollo económico, de marginalidad geográfica y social, de pobreza en cuanto a condiciones socioeconómicas y, para un sector andino, el añadido de una falta de "civilización" dada su condición de "indio". Es un diagnóstico preñado de una ideología del progreso y el desarrollo, ampliamente compartido por la burocracia estatal, los agentes políticos regionales y locales y por el conjunto de la población andina. Una conciencia más general como "andino" (referencia geográfica) e indígena (pertenencia étnica e histórica) existía como atavismo, como negación y, como tal, no tenía sentido ni podía servir como objeto de demanda ni como encuadramiento social para la acción colectiva. De este modo, no existía una percepción de pueblo, una visión de comunidad de destino basada en la historia y la cultura. Una comunidad de historia había desaparecido durante el S.XIX cuando se constituye el sistema de identidades étnicas andinas arriba reseñado; permaneciendo sólo una comunidad de copresencia, de ser andinos por la geografía que se habita. En estas circunstancias, el proyecto andino, si así pudiera llamarse, hasta bien entrada la década de los años de 1980 no es otro sino el de la integración vía asimilación (Gundermann 1997, 1998 y 1999).

¿Qué sucede, entonces, para que esta tendencia adquiera un contenido y trayectoria diferentes? Es una cuestión no detalladamente estudiada para el caso aymara, que es donde se inicia el proceso entre las poblaciones indígenas andinas chilenas. No obstante, algunos aspectos resultan bastante claros y los comentamos a continuación.

Primero, es ineludible mencionar un contexto estructural. Nos referimos al modelo de desarrollo económico basado en la exportación de materias primas que, en el caso del norte del país, se sostiene en la minería metálica (cobre) y no metálica (azufre, yodo, sales). La minería demanda ingentes cantidades de agua. Las escasas aguas superficiales existentes en esta árida zona provienen de la cordillera andina y esta es la tierra de las comunidades aymaras en Arica, Parinacota e Iquique, y atacameñas en el alto Loa y el salar de Atacama. Las aguas tenían un uso preferentemente agrícola y urbano hasta ese entonces. ${ }^{15}$ A partir de un renovado afán de captar

15 En la zona atacameña tenemos la excepción de la gran explotación minera estatal de Chuquicamata. la mina de tajo abierto de cobre más grande del mundo. la cual se aprovisiona de aguas desde fuentes en el alto Loa. 
capitales para intensificar la actividad minera ${ }^{16} \mathrm{y}$ de las oportunidades abiertas por el Código de Aguas (debido a la separación que establece entre propiedad de la tierra y propiedad del agua) para el control de aguas, se produce una intensa presión por la inscripción y titulación de ellas con fines mineros y urbano industriales. Dada la ubicación de los yacimientos mineros en sectores remotos de los Andes o en el desierto pleno sin habitantes en su vecindad inmediata, la explotación de los propios yacimientos no representó un problema para las comunidades, ${ }^{17}$ pero sí el intento, al amparo del Código de Aguas, por el control de los recursos hídricos superficiales. Se abre así un campo de potenciales conflictos entre comunidades y empresas mineras. El más importante se presentó a propósito del control de las aguas en las nacientes de la quebrada de Tarapacá, involucrando a varias comunidades del altiplano y la quebrada y a una empresa minera cuprífera con capitales mixtos chilenos y extranjeros que iniciaban la explotación de Cerro Colorado, en las inmediaciones de la comunidad de Mamiña. De este temprano conflicto (se desarrolló por varios años, hacia mediados de la década de 1980), emergió una organización de comunidades (COTALI) y el caso se transformó en una referencia obligada para lo que será un eventual movimiento aymara. Algo similar, pero con menor dramatismo, se produjo con la exportación de reproductores de llamas y alpacas, cuestión que afectaba a comunidades de ganaderos de la alta cordillera de Parinacota. Algo parecido enfrentó también a comunidades atacameñas de la cuenca del río Salado, afluente del Loa, con empresas mineras y de agua potable.

En uno y otro caso, la acción del gobierno consistió en decretar medidas administrativas tendientes a resolver los conflictos presentados sin modificar la política económica desde cuya operación surgían. Se favoreció la inscripción de aguas por las comunidades aymaras con anticipación a decretar otros derechos privados; en el de los reproductores de llamas y alpacas se introdujo medidas cuarentenarias más estrictas, previo a su exportación, de tal modo de regular el monto del flujo de exportación. Se tra-

16 Previstas por un Código Minero que otorga grandes facilidades de operación a las inversiones en el sector.

17 Salvedad sea dicha, nuevamente, de la mina Choquelimpie en la alta cordillera de la provincia de Parinacota, respecto de algunos propietarios de estancias de la comunidad homónima de Parinacota; y de Cerro Colorado en la provincia de Iquique, respecto de la vecina comunidad de Mamiña. ta de un estilo de mediación de los conflictos bajando su intensidad sin modificar un ápice de los principios más generales que los suscitan; el principal de ellos, la concurrencia expedita de los factores económicos para su libre circulación en el mercado. La ausencia de una política de protección a minorías, segmentos de población empobrecidos o grupos sociales vulnerables es apenas corregida incidentalmente ante la aparición de focos de conflicto.

Las minorías étnicas indígenas no son explícitamente reconocidas como grupos culturales con pretensiones legítimas de distintividad cultural y eventuales derechos específicos, sino como poblaciones en tránsito a confundirse con el ciudadano chileno medio de cualquier lugar del país. Esta política explícita de incorporación por asimilación se constituye como problema especialmente para los dirigentes $y$, en general, la élite indígena consciente que formula y respalda, además de demandas campesinas tradicionales, una de índole étnica. Entre los aymaras, ella venía formándose y ganando en autopercepción de sí mismos como grupo étnico con derecho a proyectarse como grupo social diferenciado ${ }^{18}$ del resto de la población nacional. ${ }^{19}$ Desde el Estado, entonces, se venía implementando un tipo general de acción que entraba en abierta contradicción con aspiraciones de continuidad étnica.

La élite aymara que protagoniza la etnogénesis reúne algunas características especiales. En primer lugar, son por lo común oriundos del altiplano; es decir, de las zonas andinas en donde la continuidad cultural indígena es mayor y en donde, como atributo crítico, existía una más definida percepción de etnicidad y de diferencia, aunque bajo la modalidad de estereotipos y estigmas. ${ }^{20}$ Luego, son por lo ge-

18 La política de asimilación y la resistencia a ella son bastante mejor conocidas que una poderosa corriente de consentimiento a la asimilación, presente en todas las minorías indígenas nacionales, en asociación con el acceso a la educación, la descampesinización, las migraciones a centros urbanos y la transformación del sistema social andino; en suma, de aceptación de la modernización. Es un tema pendiente de un mejor tratamiento en la agenda de la investigación sobre la etnicidad en Chile.

19 Cuestión que en el caso mapuche es más antigua, al igual que en la minoría pascuense, y que en el atacameño y colla se dará sólo algunos años más tarde que el momento aquí analizado (mediados y segunda mitad de la década de 1980).

20 La población de las comunidades andinas de valle se situaban por aquel entonces fuera de la condición de indígena, razón por la cual la mayoría de sus dirigentes, líderes y élite profesional no se interesó inicialmente por organizaciones y redes fuertemente marcadas por una ideología de la "indianidad". 
neral urbanos; esto es, aunque sus familias provienen de las tierras altas, su forma de vida y cotidianidad es urbana. ${ }^{21}$ Ello favorece el acceso a información muy diversificada, una familiaridad práctica de relación con una gran variedad de sujetos y ambientes (de mercado, burocráticos, de la sociedad civil, pobladores, trabajadores, coterráneos, etc.) y una capacidad de desenvolvimiento relativamente alta para la gestión de sus intereses y propósitos. Además, un cierto número de ellos son técnicos o profesionales con formación universitaria. Destacan entre los primeros los contadores y entre los segundos los profesores de enseñanza básica más tarde la incorporación de otros profesionales jóvenes que van saliendo por aquel entonces de la universidad tendrá lugar en el contexto de la acción de la Corporación Nacional de Desarrollo Indígena (CONADI), un organismo estatal a la cual los principales miembros de esta élite se incorporan.

El estigma, al modo de un substrato de conciencia étnica, primero, y el contacto con canales de comunicación más globales en las instituciones de educación media, técnica y superior, lo cual les permite acceso a la historia, al discurso indigenista e indianista y el contacto con sensibilidades neo románticas, después, representa la prehistoria de la asunción de una identificación aymara positiva y la definición de demandas étnicas. El marxismo como ideología se encuentra en la década de 1980 en crisis, y no sólo en la izquierda política, sino que también en medios, como los universitarios, donde antes ésta tuvo particular fuerza. No representaba el marxismo, entonces, una opción ideológica prestigiosa. Por lo demás, a diferencia de los mapuches, entre aymaras y atacameños la izquierda o las ideas marxistas nunca se vincularon con lo indígena, salvo quizá como condición a superar, debido a que no existía una tradición de organización de izquierda andina y las ideas de la izquierda política eran en la región definitivamente contradictorias con una valoración y recuperación de lo indígena (visión cla-

21 Con una que otra excepción, los dirigentes rurales no ayudan a formar o no se incorporan en la red de relaciones que vincula los miembros de la élite aymara entre sí. Ello tendrá lugar mucho más tarde, durante la década de 1990. Escapa de las posibilidades de este trabajo un análisis de esta no participación y, en general, de la considerable desvinculación entre los iniciadores de un movimiento y bases urbanas populares $o$ campesinas. Digamos tan sólo que ello no representa un tema nuevo en los estudios sobre etnogénesis y, de manera general, en las investigaciones sobre movimientos étnicos y nacionalismos. sista de la sociedad, internacionalismo proletario, ideas de dinámica histórica como progreso, etc.).

Ya no en el nivel de los actores, sino en uno estructural, se puede recordar que quienes constituirán la élite étnica, por aquel entonces apenas en formación, son parte de un estrato de población aymara en proceso de movilidad social. Esta movilidad social encuentra considerables obstáculos para realizarse, debido a lo cual el bloqueo a la integración con movilidad social puede considerarse también un factor que otorga la posibilidad de un punto de cambio desde una identificación con una ideología de la modernización (cambio, ascenso social, progreso, adelanto, seguridad económica y social, etc.), hacia identificaciones más complejas que hacen prevalecer la aboriginalidad, indianidad, andinidad y aymaridad. Es decir, introduciendo a la dinámica del cambio andino una ideología de tradicionalidad reprocesada ("invención de la tradición") como dimensión de proyección colectiva. Al interior de la sociedad aymara y en las condiciones reseñadas, por tanto, se hacen más heterogéneas y complejas las formas de conciencia social y en ellas una perspectiva étnica positiva parece llegar para quedarse por largo plazo (González y Guerrero, 1990).

Pero, lo anterior no es lo único que concita lả constitución de redes y de organizaciones de una élite con clara conciencia y propósito de acción colectiva según un eje de etnicidad. La red de individuos y organizaciones logra movilizar recursos importantes en su favor. Por lo pronto, intelectuales de la región, que habían investigado o que investigaban sobre ella y que son responsables de un hecho crucial: provistos de la legitimidad del discurso académico, a través de sus publicaciones y participación pública ${ }^{22}$ contribuyeron de una manera decisiva a instalar en la conciencia nacional y también en la de la élite indígena en formación, una nutrida información y representaciones acerca de lo aymara y lo atacameño. Contribuyen a la elaboración de una imagen de "indio" no estigmatizada, sino que más bien víctima de una historia de dominación, a la vez que envestida de la dignidad de una cultura propia.

No es desdeñable, asimismo, la existencia de una sensibilidad ecológica bastante extendida que, por

22 Actividades de extensión académica, participación en ONGs, participación política, comunicación con círculos sociales y políticos regionales y nacionales, relaciones formales e informales con dirigentes, intelectuales y organizaciones andinas 
asociación con la difundida imagen del indígena en sintonía con conducta ecológica, granjeó muchas simpatías para las ideas y pronunciamientos de dirigentes y organizaciones. Hacia finales del período autoritario se da una feliz convergencia, entonces, entre discurso de los indígenas y valores ecológicos. Ello permitió a las organizaciones y a sus miembros más activos contar con una buena recepción y cobertura en los medios de prensa. Ellos mismos ganaron conocimiento y confianza en el manejo de los medios de comunicación, percibiendo con claridad su gran importancia. La exportación de reproductores de llamas y alpacas, pero sobretodo la eventualidad de extracción de las aguas del lago Chungará con fines agrícolas y de consumo urbano de Arica, fueron dos eventos en los que un gran público regional y nacional puso sus ojos sobre la geografía andina, sus recursos y sus habitantes, los aymaras. Con ello, se les conoce y se les reconoce por círculos progresistas y corrientes políticas que más tarde tendrán activa participación en la Concertación y el gobierno de la transición. ¿Quienes podrían ejercer interlocución? Ciertamente no los pastores andinos de la alta cordillera con sus llamas, sus riachuelos, sus vegas, sus llamas y sus capillas características. Podrían, quienes contaban con conocimientos, experiencia y argumentos que encontraran eco en públicos generales o específicos y estratégicos a mediano plazo. ${ }^{23}$

En la vera de esa misma corriente de solidaridad, cuentan con el apoyo de organismos no gubernamentales regionales y nacionales, quienes canalizan hacia ellos información, vínculos a redes alter-

23 Es notable que a partir de mediados de la década de 1980 la instalación y las operaciones de la mina de oro y plata de Choquelimpie, con un considerable potencial de contaminación (operaciones con cianuro, captaciones de agua subterránea importantes, etc.), no fuera objeto de una reacción en los medios y en las organizaciones que guardara equivalencia o proporción respecto de otros eventos como el del lago Chungará, a escasos kilómetros de Choquelimpie en la misma área de Parinacota. Esto habla de la condición coyuntural, hasta cierto punto, del reconocimiento de "lo andino" en la esfera pública y política. Pero, también, de la visibilidad de los problemas y amenazas sobre lo andino. Condiciones mejores (situación política nacional en rápida evolución, acumulación de información y difusión de lo andino, generación de una base de organizaciones con objetivos étnicos, elaboración de discursos, etc.), para esa emergencia se dieron ya hacia finales de la década y no antes como lo sugiere la pasividad general respecto de Choquelimpie. Se debe reconocer que una política acertada de la empresa en el manejo de su proyecto favoreció también mucho esa relativa indiferencia. Desde luego, hoy sería impensable esa ausencia casi total de reacción. nativas y círculos políticos, servicios y algunos recursos financieros para el desarrollo de actividades conjuntas o autónomas. También, con la Iglesia Católica a través de los Obispados de Arica, Iquique y la Prelatura de Calama con sus Departamentos de Acción Social (DAS), los cuales a su vez canalizaban recursos provenientes de una ONG con alcance nacional perteneciente a la Iglesia y llamada Oficina de Capacitación y Asistencia Campesina (OCAC), muy exitosa en obtener apoyo financiero internacional y bastante eficiente también en distribuirlo hacia las clientelas y conexiones campesinas e indígenas de los obispados. Difícilmente se podría entender el éxito de estos intelectuales, dirigentes y organizaciones, durante la década de los años de 1990, en concentrar a través de ellos las piezas maestras de la política del Estado respecto de los indígenas en el norte del país, si no se toma en cuenta, entonces, la movilización de recursos intelectuales, institucionales y financieros que lograron concitar o que se les prestó solidariamente entre 1988 y 1990.

Una ONG, la Comisión Chilena de Derechos Humanos, a través de un conjunto de reuniones organizadas en el centro y sur del país, ${ }^{24}$ busca elaborar una Propuesta Constitucional de los Pueblos Indígenas, procesable políticamente durante el retorno a la democracia y con base en el triunfo de la oposición sobre la derecha política y al poder militar en elecciones libres. Su acción, en coordinación con las organizaciones aymaras que se habían formado o estaban en formación, a las cuales se les une tardíamente una atacameña, y con las ONGs regionales permitió algo inédito: que aymaras y luego atacameños, junto con dirigentes y organizaciones rapanuis y mapuches se conocieran, dialogaran, establecieran vínculos y acuerdos, organizaran declaraciones, se involucraran en los temas étnicos con alcance nacional hasta ese momento concentrados en los mapuche. Las cuestiones de la autonomía, los territorios (y no simplemente la propiedad) y el

\footnotetext{
24 Tenemos información de tres de ellas. La primera se realizó en Temuco, corazón de la región mapuche (4-6 de agosto de 1988), con la participación de Aymar Markas de Iquique; la segunda en Niágara, también en la zona mapuche, en noviembre de 1988, y la tercera en El Canelo de Nos, Santiago (2022 de enero de 1989), en la que se encontraban presente delegados de la Federación de Organizaciones Aymaras, la que incluía por ese entonces a nueve de ellas.
} 
reconocimiento como pueblos en la Constitución son ahora los temas cruciales. La demanda indígena andina se proyecta desde una modulación campesina hacia una étnica regional (aguas, camélidos, etc.), y de esta hacia una nacional compartida con las demás etnias indígenas del país.

Una proyección fundamental la obtiene esta élite desde la Concertación de Partidos por la Democracia como coalición de partidos de oposición a la dictadura de Pinochet y los militares, en momentos en que se plantea una posible apertura hacia el retorno a la democracia. Debido a la relación existente entre la oposición política y las organizaciones e instituciones que facilitaron la movilización de recursos resumida, las organizaciones indígenas, las andinas entre ellas, pudieron de manera expedita establecer comunicación y una activa discusión con la Concertación en la eventualidad, cada vez más plausible a medida que pasaban los meses, de su acceso al gobierno; todo ello bajo la consideración de ser un grupo especial del país que requería un también especial tratamiento de sus problemas. Los hitos temporales de este proceso están señalados por el plebiscito convocado por los militares en 1998 para decidir la continuidad del régimen autoritario y, más tarde, con el inesperado fracaso de este ejercicio de manipulación política, el llamado a elecciones competitivas para formar gobierno (a finales de 1989). En esta coyuntura y por razones que son más contingentes que necesarias, el tema de los pueblos indígenas es tomado por la Concertación (junto con el de mujeres, los pobres, y el medio ambiente) como expresión de temas especiales. De esto surge el Acuerdo de Nueva Imperial, suscrito en esa localidad de la región mapuche el 1 de diciembre de 1989 por Patricio Aylwin. El acuerdo vino a ser la ratificación de una propuesta hecha por la Concertación a los pueblos indígenas el 12 de octubre de 1989, la cual surgió desde la comunicación que pudieron establecer previamente los pueblos indígenas con el candidato y los dirigentes de la Concertación a partir de la mediación de entidades de la sociedad civil que trabajaban o se vinculaban con las organizaciones indígenas (Iglesia, ONGs, intelectuales, etc.), o al menos con su activo concurso. En el acuerdo de Nueva Imperial el entonces candidato a la presidencia por las fuerzas políticas democráticas y futuro presidente de Chile se compromete a la creación de una Comisión Especial de Pueblos Indígenas con participación paritaria de representantes de pueblos indígenas. A esa comisión se le asignará la tarea de proponer el reconocimiento constitucional de los pueblos indígenas de Chile y de promover una legislación que favorezca su desarrollo; el instrumento de lo anterior será la Corporación Nacional de Desarrollo Indígena (CONADI).

La Comisión Especial de los Pueblos Indígenas (CEPI) se crea por decreto supremo el 17 de mayo de 1990, a pocas semanas de haber asumido el gobierno de la Concertación. Dos consejeros aymaras se incluyen en la nómina, ambos miembros importantes de organizaciones urbanas (Pacha Aru, de Arica, y Aymar Markas, de Iquique) que habían participado en los dos años anteriores en el proceso que condujo a la alianza de estas organizaciones con la Concertación. La designación de un Consejero atacameño ligado a la organización Likan Kunza será posterior. Esos mismos consejeros ocuparán luego los más altos cargos de la Subdirección Nacional de CONADI en el norte del país, con la inclusión de algunos de sus compañeros de las organizaciones desde las que surgieron.

Podemos recapitular lo dicho hasta aquí recalcando que luego del surgimiento de intelectuales, profesionales y organizaciones que desde mediados de la década de 1980 se plantean una demanda de tipo étnico, se da curso a un proceso complejo, ambiguo e incluso contradictorio de relaciones, producción de acuerdos, pactos, y también discensos, tensiones y puntos de conflicto entre las principales organizaciones indígenas del país vigentes por aquel entonces, andinas incluidas (varias aymaras y una atacameña hacia el final de estos años), y la coalición política triunfante de la derrota política de la dictadura. En ese decurso, un factor eficiente es la movilización de recursos que esas organizaciones logran convocar y que también se les ofrece desde la sociedad civil (Iglesia, ONGs, intelectuales), la cual mantenía una fluida relación con los dirigentes y corrientes partidarias en la oposición. Esa mediación cesa con la creación de la CEPI. Desde ese momento, el vínculo de las organizaciones andinas, los partidos políticos y el gobierno de la Concertación se realiza directamente.

\section{El pacto entre Concertación de Partidos por la Democracia y aymaras, atacameños y collas}

El movimiento indígena chileno está lejos de ser unitario. La relación con la Concertación y con los gobiernos de Aylwin y Frei tampoco ha sido unifor- 
me. ${ }^{25}$ Hacia finales de la década de 1980 las organizaciones aymaras se diferenciaban entre sí sobre la base de discursos e ideologías, ${ }^{26}$ tipos de liderazgo, ${ }^{27}$ tipo de miembros, ${ }^{28}$ vínculos con población de base ${ }^{29}$ y estrategias de trabajo. ${ }^{30}$ También ellas tensaron internamente su unidad y con la Concertación desde muy temprano. La aventura del Partido por la Tierra y la Identidad (PTI) es el mejor ejemplo de lo anterior.

En marzo de 1989 sale a la luz pública el Partido de la Tierra y la Identidad, encabezado por un grupo de dirigentes mapuches y aymaras. Prácticamente toda la élite de aymaras que en años anteriores habían incorporado y elaborado para las circunstancias regionales un discurso étnico (con influencia indianista, como se lleva dicho) se involucró en el PTI. No ocurrió lo mismo en el caso de los mapuches, desde donde surgieron voces muy críti-

25 La heterogeneidad es hoy día un dato relevante de la causa si advertimos que en el conflicto mapuche son organizaciones que se encuentran fuera del círculo de acción de la CONADI (Consejo de Todas las Tierras y La Coordinadora Arauco Malleco), las que protagonizan el movimiento mapuche (Foerster y Vergara 2000).

26 Fuertemente indianista en el Pacha Aru de Arica; desarrollismo sobre bases local comunitarias en el caso del Centro Cultural Aymara; desarrollismo sobre una base tradicional más heterogénea en el de Aymar Markas.

27 Más carismáticos en Pacha Aru, relativamente más democráticos en las restantes organizaciones.

28 Estudiantes universitarios y jóvenes aymaras urbanos en Pacha Aru, comunidades y campesinos de comunidades en el Centro Cultural Aymara, comunidades y pobladores urbanos migrantes en el caso de Aymar Markas.

29 Inexistentes o bastante tenues en Pacha Aru; algo más desarrolladas en las restantes organizaciones. En el contexto del gobierno autoritario y con el temor a alguna forma de castigo, las relaciones entre estas organizaciones que se encontraban fuera del circuito de las organizaciones de base territorial oficiales y asociaciones también surgidas desde iniciativas del gobierno, los compromisos efectivos entre organizaciones étnicas y comunidades eran en los hechos inexistentes; es más realista decir que existían alianzas provisorias, poco consolidadas y no públicas con personalidades, grupos o facciones de algunas comunidades andinas rurales. La acción concertada y unificada fue una excepción, no la regla.

30 Muy centrada en la re-etnificación o inculcación doctrinaria del Indianismo, con cierto éxito entre estudiantes y jóvenes aymaras urbanos de Arica y con mucho menos llegada a otras categorías de edad y a campesinos, en el caso de Pacha Aru; planteada en torno a la defensa y captación de recursos para desarrollo de base en el Centro Cultural Aymara; modalidades de acción asistenciales y de promoción cultural en el de Aymar Markas. A lo anterior se añaden esfuerzos compartidos de coordinación, esfuerzos de federación, alianzas estratégicas ante las ONGs, etc., normalmente de corta duración y atravesadas por tensiones, aun cuando exitosas en crear un espacio, un discurso y formas de acción con sentido étnico. cas. Tampoco hubo participación atacameña digna de mención. El partido es concebido como "un instrumento para asegurar la sobrevivencia de los Pueblos Indígenas", ${ }^{31}$ por medio del cual se aspira a ganar representación parlamentaria autónoma de los partidos, el reconocimiento constitucional como pueblos, el fomento cultural, la protección de los recursos que las etnías aún controlan y el desarrollo con autonomía. A despecho de un análisis más pormenorizado aún no realizado, señalemos dos aspectos indispensables a la hora de comprender el paso dado por este grupo de aymaras y algunos mapuches. Primero, la profunda desconfianza en el sistema político, los partidos, sus programas y su voluntad de incorporar los temas indígenas como algo más que un problema sectorial menor. Por aquel entonces no existía todavía el acuerdo de Nueva Imperial que le daba una visibilidad particular a la temática indígena $\mathrm{y}$, de otra parte, el sistema de partidos estaba reconfigurándose bajo las reglas que lograron negociarse con la dictadura, lo que permitiría un espacio político posible a una iniciativa indígena de levantar una representación parlamentaria independiente. Por otra parte, la ideología indianista que profesaban los intelectuales aymaras y algunos mapuches, influidos por el Consejo Indio Sudamericano (CISA) y el discurso de los partidos indios bolivianos que influyeron a algunos aymaras chilenos, además de argumentos de autonomía incorporados y re-elaborados desde los foros políticos y del derecho internacional, ${ }^{32}$ así como, de manera más general, sensibilidades neo-románticas, hacen a lo menos problemática una aceptación irrestricta de la representación política por cauces partidarios convencionales.

El fracaso de esta iniciativa se consumó en las elecciones parlamentarias de finales de año. Los errores de cálculo electoral fueron mayúsculos; ${ }^{33}$ otros tantos se cometieron al creer en una identificación masiva de la población aymara con estas ideas y propuestas $^{34}$, y ni hablar de las decisiones de apoyo

31 Partido de la Tierra y la Identidad. Declaración de Principios (1989:2-3.)

32 Lo que según otra lectura significa rebajar o decididamente poner en cuestión la legitimidad del modelo del Estado nación monoétnico y las estrategias de gestión política de intereses colectivos tradicionales en ese marco.

33 Se sobrestimó el número de electores. Se consideró una población aymara de 100-120.000 personas, de las cuales 5060.000 serían electores, suficientes como para conseguir una elección senatorial.

34 En la región se consiguió alrededor de 2.000 votos. 
a candidatos. ${ }^{35}$ No obstante, conservaron la cordura suficiente como para mantener la comunicación y diálogo con la Concertación para la futura gestión de lo indígena, ante la segura eventualidad de que el ganador de las elecciones presidenciales fuera el candidato de este conglomerado de partidos. Su participación en el acuerdo de Nueva Imperial es indicativo de lo anterior, bajo el supuesto del apoyo electoral indígena a la Concertación en la elección presidencial, ya que no en la de diputados y senadores. ${ }^{36}$ Una organización partidaria autónoma no es un tema clausurado y se mantiene como una posibilidad que espera mejores tiempos. Esto le da a la alianza indígena con la Concertación, por este y sin duda también por otros motivos, una condición inestable.

Desde las elecciones generales de finales de 1989 y el fracaso de su gestión autonomista, las organizaciones y dirigentes aymaras activan el desarrollo de relaciones y el alineamiento con la Concertación. El costo que pagaron por la inexperiencia y la confusión fue menor de lo que pudo ser. La tabla de salvación provino del Partido por la Democracia (PPD), un partido nuevo, progresista, con principios generales afines a una social democracia y con una composición de miembros, grupos e intereses bastante heterogéneos. El grueso de la renovación socialista se afilió en este partido y en este sector es donde la temática de los pueblos indígenas, como el de las mujeres y el medio ambiente, encontró una acogida especialmente favorable. Ello significó que en el período comprendido entre las elecciones presidenciales, de senadores y de diputados, la creación de la CEPI y la promulgación en 1993 de la Ley $N^{\circ} 19.258$, también llamada Ley Indígena, que estipula la formación de la Corporación Nacional de Desarrollo Indígena (CONADI), los dirigentes aymaras principales se afiliaran al PPD y, en lo sucesivo, la CONADI fuera considerada en el norte

35 Contradiciendo todo lo postulado, se apoyó en la región administrativa y electoral senatorial de Tarapacá al abogado Fernando Dougnac, quien en años anteriores había defendido legalmente, a partir de su contratación por organismos vinculados a la Iglesia Católica, a comunidades aymaras y atacameñas amenazadas por la aplicación del Código de Aguas. Este mismo señor se permitía en la campaña hablar de "mis aymaras", recordando al más conservador de los hacendados de la zona central de Chile respecto de sus peones e inquilinos entendidos, cuando pudieron votar, como masa electoral cautiva disponible para sus aventuras políticas.

36 Cuestión que, para complicar más las cosas, posteriormente no se materializó en una votación favorable a la Concertación en los distritos electorales indígenas aymaras. del país un organismo público cuyo cupo político correspondía al PPD. Entre tanto, sin mayor éxito, la Democracia Cristiana, el principal partido de la Concertación, buscaba en el norte del país crear una base política entre los dirigentes aymaras de base y algunos profesionales afines.

La unidad indígena en el país es exclusivamente coyuntural o estratégica. La presión que los líderes y las organizaciones mapuches imponen sobre el gobierno no tiene parangón con la de las demás etnias, las andinas incluidas. Otro tanto se produce con la representación mapuche mayoritaria en el Consejo Nacional de la CONADI (un órgano paritario organizaciones indígenas - Estado), donde los consejeros y representantes del gobierno de ascendencia mapuche suelen representar la mayoría. Esto no siempre fue así. De hecho, hubo momentos a principios de la década de 1990 en que se manifestó una considerable unidad. Aunque se inició con anterioridad a la creación de la Comisión Especial de los Pueblos Indígenas (CEPI), es en torno a la gestación, elaboración, tramitación y promulgación de la Ley Indígena que se expresó un mayor grado de unidad. El bloqueo a iniciativas propias para favorecer a los mapuche y la mínima representación y poder de los pueblos andinos y el rapanui en el Consejo $\mathrm{Nacional}^{37}$ como para hacer prevalecer mociones de su interés, junto con una percepción de desigualdad notoria en las partidas presupuestarias, todo ello condujo en definitiva a una animosidad manifiesta contra las dirigencias mapuches, cuestión que permanece hasta el día de hoy. ${ }^{38}$

37 El llamado Segundo Acuerdo (el primero fue con la Concertación y suscrito en Nueva Imperial), fue alcanzado en la localidad de Niagua el 27-28 de enero de 1990, en vísperas de la asunción del nuevo gobierno. Incluye un mayor número de grupos indígenas (aproximadamente los mismos con los que tratará más tarde la CONADI: aymara, mapuche, rapanui, huilliche, pehuenche --estos dos últimos segmentos del pueblo mapuche--, kawashkar y atacameño). El acuerdo considera la creación del Consejo Nacional de Pueblos Indígenas de Chile, incluyendo como comisión ejecutiva a la Comisión Técnica de Pueblos Indígenas de Chile, entidad que surgió con el Proyecto de Reconocimiento Constitucional de los Pueblos Indígenas al amparo de la Comisión Chilena de Derechos Humanos. Representa un esfuerzo de representación nacional ante el triunfo de la oposición y ante la situación de apertura que se abría con el gobierno de la Concertación.

$38 \mathrm{Al}$ punto que sistemáticamente se intentan estrategias de minoría en el Consejo nacional, entre aymaras ( 1 consejero), atacameños (I consejero) y rapanuis ( 1 consejero) contra los mapuches 
La Ley $\mathrm{N}^{\circ} 19.253$ fue promulgada en 1993 después de una larga tramitación, aunque finalmente con una aprobación unánime en ambas cámaras. ${ }^{39}$ No se logran el reconocimiento y la autonomía, las dos cuestiones centrales defendidas por las organizaciones, líderes e intelectuales más radicales. En subsidio de ello se habla en el cuerpo de la Ley de "poblaciones" (y no de pueblos) y de "áreas de desarrollo" (no de territorios); se rebaja al mínimo, entonces, el reconocimiento y los derechos sobre el territorio y sus recursos. Se logra una institucionalidad (la CONADI), y una política definida como "de desarrollo con identidad". Es decir, que por parte del Estado y del gobierno de la Concertación se establece un compromiso de reorientar una política ya centenaria de asimilación cultural hacia el respeto y el fomento de la distintividad de los indígenas como grupos humanos con derecho a perseverar en sus valores, creencias y costumbres. Se asume el compromiso, asimismo, de dar un impulso mayor que el hasta ahora realizado por los gobiernos al desarrollo de una población masivamente pobre (acceso a servicios básicos, educación, fomento productivo, asistencia social, etc.).

Los dirigentes e intelectuales aymaras de las organizaciones que participan en este proceso terminan por aceptar la obtención de una ley que se promulga con recortes y rebajas significativas. Se la acepta como un paso adelante, como una base mejorada de trabajo para la obtención de objetivos étnicos más profundos, de más vasto alcance y a más largo plazo. Sus principales exponentes se incorporan a la Subdirección Norte, la que cuenta con su sede en la ciudad de Iquique (capital de la región de Tarapacá) y dos oficinas provinciales: una en Arica para Arica y Parinacota y otra en San Pedro de Atacama (en los hechos Calama), para la provincia de El Loa y los atacameños. Lo hacen en calidad de funcionarios públicos. Ya bien avanzada la década de 1990 se crea una oficina para los collas en la ciudad de Copiapó y dependiente de la Subdirección Nacio-

39 Por lo demás, una rareza ya que sólo en contados casos la promulgación de una ley alcanza en Chile cuorum tan calificados. No es el resultado de una especial sensibilidad de la clase política hacia los indígenas, sino que un mero reconocimiento a los severos problemas sociales que arrastran estas poblaciones. Con excepciones, persiste una mirada tradicionalizante. folclorizante, etnográfica y carencialista en la clase política. Un buen síntoma de esto es la negación tajante que se mantuvo durante la tramitación, tanto en la derecha como en la Concertación, para el reconocimiento como pueblos y el control de territorios y recursos, las dos cuestiones claves de la propuesta indígena. nal Norte. Recientemente, se forma otra en Putre para la atención de los aymaras de la provincia de Parinacota.

De manera un tanto optimista, inicialmente, se valora quizá en exceso las ventajas que ofrece una instalación institucional, mandatos legales y recursos provistos por la Ley Indígena. Se debe recordar, por una parte, que se trata de líderes e intelectuales que venían por largos años realizando una entrega de trabajo comprometido y solidario en el cual, a pesar de contar usualmente con algún financiamiento para sus actividades, la posibilidad de remuneraciones estables desde el Estado les representaba una seguridad y posibilidad de proyección para sus proyectos individuales y familiares largamente postergados. Por otra parte, la llegada a la CONADI no se tradujo en un poder actuar en lo sucesivo desde una plataforma de trabajo expedita y potenciada, como en momentos iniciales se pensó; por el contrario, enfrentaron problemas de gran envergadura, muchos de los cuales hasta ahora no se han resuelto. Con el arribo a la CONADI se plantearon una salida realista al dilema entre consecuencia con sus principios y objetivos, que la Ley Indígena desechó o incorporó sólo de manera tibia, y el quedar al margen del Estado, en una posición de marginalidad como la que se tenía a inicios de la década de 1980. A tono con el pragmatismo aymara se adoptó una salida de compromiso. Esta podría resumirse en lo siguiente: actuar desde dentro del Estado en función de objetivos étnicos de más largo plazo, exteriores y eventualmente contradictorios con la política étnica que demarcó la Ley Indígena. Quedarse fuera, se pensó, significaba permanecer en la marginalidad o en un tipo de protagonismo confrontacional, más próximo al del actual movimiento mapuche, cuestión que no se compadece con sus estrategias y, de manera más general, con la cultura política legalista, realista, flexible y negociadora aymara.

Se trata de una opción con algunas ventajas, pero también con un alto costo. Desde el punto de vista de un movimiento, el principal de ellos es la cooptación, el tener que actuar con arreglo a procedimientos, objetivos y alcances que no son los de las organizaciones; en una palabra, la pérdida de autonomía. La opción por una base institucional mejorada para una acción de largo plazo entra en contradicción, o por lo menos genera una permanente tensión, con la política indígena del gobierno 
de la Concertación. Esto provoca un doble estándar: un rol de burócratas, por una parte; uno de intelectuales y líderes indígenas, por otro. ¿Qué resultó de todo esto? Intentemos un balance preliminar.

\section{La Corporación Nacional de Desarrollo Indígena es} un organismo sectorial cuyo mandato es el de coordinar y promover el etnodesarrollo entendido en los términos que lo estipula la Ley Indígena: desarrollo con identidad. La acción de la Subdirección Norte y sus oficinas dependientes por casi ocho años no arroja, según nuestro entender, un balance positivo claro. En primer lugar, no hay un cambio en las sociedades andinas regionales visible atribuible a su acción. La continuidad de los valores de diversos indicadores sociales generales así lo estipula, ${ }^{40}$ a pesar de que, en términos generales, las áreas andinas gozan de asignaciones presupuestarias especialmente altas en proporción a la población que allí habita. Hay cambios de cierta importancia producidos en la década, pero ellos podrían atribuirse a dinámicas internas de desarrollo y a la acción de otros organismos del Estado (Instituto de Desarrollo Agropecuario, Dirección de Riego, Dirección de Vialidad) y a los gobiernos municipales (en las áreas de educación, salud e infraestructura local), más que propiamente a la CONADI. Esta ha contribuido lo suyo (becas de estudio, proyectos de fomento productivo), pero sin destacar como un agente inductor eficiente del desarrollo. Tampoco fue capaz de alcanzar una cobertura satisfactoria de acción hacia la población indígena urbana, mayoritaria en sus respectivas etnias, las cuales siguen sistemáticamente fuera de sus capacidades de identificación y definición de acciones. Avances todavía modestos, particularmente en Arica, ha logrado en su posicionamiento dentro del Estado. Sus atribuciones de ente coordinador de acciones hacia los indígenas ha fracasado hasta ahora ${ }^{41}$ En la disputa por el control de recursos, la creación y el control de clientelas, las

40 Pobreza, satisfacción de necesidades, etc., de una población que se encuentra tipificada entre las más pobres del país.

41 Ya por demasiado tiempo se habla de CONADI en la región como "con nadie"; es decir, aislada, sin ninguna capacidad de incidir sobre la acción hacia los indígenas. Esta incapacidad se amplía hasta incluir los gobiernos municipales quienes, en parte por razones políticas (algunos municipios con población indígena mayoritaria son controlados por alcaldes de derecha), y en parte por el débil reconocimiento de la propia institución (legitimidad, aceptación de autoridad), no tienen interés para actuar de consuno con CONADI. La disputa por recursos, la competencia por clientelas, la lucha por el posicionamiento dentro del campo burocrático son también factores intervinientes. pugnas por el poder y la legitimidad dentro del campo burocrático regional, la CONADI ha logrado sólo el dudoso estatus de la supervivencia.

Logros de mayor interés son anotables si consideramos los objetivos que la élite que entra a controlar la CONADI regional se planteó en cuanto integrantes y cabecillas históricos de un movimiento. En primer lugar, un reconocimiento y legitimidad no endosable al hecho de que desde la CONADI actúa sólo el Estado, sino que también lo hacen personas indígenas que guardan un compromiso con la situación y el futuro de la población indígena, y que a la vez son o fueron dirigentes y líderes de organizaciones aymaras respecto de las cuales se mantenía membresía y compromiso. Amplían de este modo una legitimidad, un reconocimiento con el que no contaban, salvo en las propias organizaciones, básicamente urbanas, pequeñas, poco conocidas y con problemas de aceptación entre la población rural. ${ }^{42}$ Segundo, la creación de una red clientelar compuesta por las organizaciones de origen, organizaciones profesionales aymaras que surgen al amparo de la decisión de favorecer y fortalecer a profesionales y técnicos propios y organizaciones nuevas que toman la forma de comunidades y asociaciones indígenas. ${ }^{43}$ Esta red representaría a la vez una base de trabajo, un garante desde el cual obtener legitimidad para su acción y una red desde la cual irradiar una visión de lo andino y una propuesta de construcción de sociedad. La crisis y el eventual término de la opción de trabajar "desde dentro" del Estado se plantea, precisamente, cuando se fisura esta red clientelar. Tercero, un posicionamiento político regional a partir de su militancia en el PPD. Se trata

42 Donde, por lo demás, ya existía una nutrida red de organizaciones locales. Donde, también, la identificación como indígena fue problemática y lo sigue siendo, debido a la resistencia ofrecida por las poblaciones de los valles para una identificación a la cual se sentían y en parte sienten ajenos, según se anotara más arriba.

43 Las cuales corresponden a las figuras jurídicas contempladas por la Ley Indígena. Contra lo previsto, las comunidades andinas (bajo la forma de sucesiones en las tierras altas y de comunidades locales en los valles precordilleranos) no buscaron el reconocimiento como tales a partir de esta figura legal (un aspecto crítico de ello es el rechazo a formas comunitarias de propiedad y a las restricciones de la propiedad que introduce la Ley Indígena para la tierra y los recursos indígenas), por lo que ambas personerías jurídicas han servido hasta ahora para ampliar el abanico de recursos jurídicos ya disponibles con los cuales grupos, familias, facciones, asociaciones, etc., se dotan de una figura legal que favorezca la gestión de recursos, canalizar requerimientos, fortalecer solicitudes ante el Estado, etc. 
de una militancia instrumental en función de actuar hacia lo indígena desde el interior del sistema de partidos en el gobierno y con las ventajas que esto proporciona. Aun cuando no se ejercen mayores presiones doctrinarias sobre ellos, esa pertenencia partidaria se traduce en restricción para una acción autónoma: la de estos individuos ahora en el Estado y la de su red debe mantenerse dentro de los márgenes de maniobra planteados por la ley indígena y las reglas básicas de operación de las instituciones del Estado. Ese posicionamiento no ha estado exento de problemas y crisis. Se encuentran con un ambiente político electoral adverso entre la propia población andina que ha venido en una alta proporción negando su adhesión a la Concertación. Obviamente, las miradas se dirigen a la CONADI y se levantan preguntas acerca del efecto de su actuar, de la pertinencia de la asignación de recursos en el medio indígena y de la propia acción del grupo de elite para ganar adhesión entre su propia población. Cuarto, la masificación de un lenguaje de la etnicidad. Se instala el discurso de la etnicidad, que no debe confundirse con la ideología indianista, la cual queda reservada a los miembros de la élite, algunos profesionales e intelectuales; es decir, radicada en un medio urbano. Sí se irradia e incorpora el de la cultura andina, el del reconocer la cultura aymara como una realidad regional visible; sí gana terreno el argumento del derecho moral y legal a la continuidad cultural; sí se avanza en el reconocimiento de las raíces indígenas, sin que ello sea visto del mismo modo que antes, como extranjeridad o inferioridad. Lo étnico entra al lenguaje de los burócratas y los políticos y al sentido común de la población de una manera inédita por su profundidad y extensión. De todos modos, en la propia población andina versiones más intelectualizadas y doctrinarias de una ideología indianista, así como sus implicaciones programáticas, no corren con suerte hasta ahora. Un proceso similar se vive en la zona atacameña. $^{44}$

El grupo de élite en la CONADI a la vez favorece y potencia la acción de las organizaciones étnicas. Estas gozan de un espacio de acción distendido en el Estado, o por lo menos considerablemente más amplio que en tiempos de la dictadura. Los recursos fluyen, se pueden multiplicar acciones, el acceso extensivo a la población indígena en sus distintas localizaciones es ahora plausible, etc. Se cuenta con amparo político en la coalición de gobierno. El costo es, a falta de un término mejor, la domestica- ción de un movimiento incipiente. En los términos de la teoría de los movimientos sociales, la dinámica de la acción de las organizaciones no se desarrolla en la dirección de la ruptura de los límites de compatibilidad del sistema (Melucci 1996, 1998); aquella se mantiene al interior del sistema, básicamente desmovilizada como movimiento. La acción en curso es desarrollista y congruente con la política indígena estatal. Algo muy distinto ha venido ocurriendo con los mapuche, como ya sabemos.

Desde las organizaciones de base, ${ }^{45}$ la CONADI representó poco más que un organismo especializado del Estado, donde pudieran ser mejor acogidos o suplementariamente atendidos. Se desvanece rápidamente la ilusión de algunos dirigentes de encontrar un espacio de acogida con el cual se pudiera dialogar con el Estado en los términos de un mismo código de comprensión de la acción. Pese a la resistencia que se ofreció y en parte por los errores de manejo financiero y de gestión que se cometieron, se les ha venido imponiendo cada vez más rígidos formatos de administración, asignación y evaluación de recursos y servicios. En verdad, la relación con las bases nunca pudo plantearse de un modo diferente al de una burocracia estatal. En esta relación, además, compitieron por la constitución de clientelas con un éxito limitado. Luego de los primeros años, la posibilidad de dar cabida a una acción simultánea de doble faz (burocrática y étnica) se tornó cada vez más insostenible para el grupo que controlaba CONADI. Se debió optar por algún tipo de solución. Circunstancias externas los empujaron también hacia una salida híbrida. De ella surge el Consejo Nacional Aymara y la democratización de la elección de Consejeros Nacionales ante la CONADI.

\section{Despejando la dualidad y desarrollando una po- sición de autonomía frente al gobierno: la acción del Consejo Nacional Aymara como fenómeno emergente}

Con la promulgación de la Ley Indígena surge la tarea de crear, organizar y poner en funciones la Subdirección Norte. Como ya se ha insistido, la di-

44 En el caso colla, su pequeño número, débil organización y ausencia de intelectuales los deja fuera de estas consideraciones.

45 Comunidades, asociaciones, juntas de vecinos, grupos religiosos, grupos solidarios, agrupaciones deportivas, organizaciones gremiales, etc. 
rección y algunos cargos fueron cubiertos con los miembros más prominentes de las organizaciones de corte étnico reivindicativo que desde finales de la década anterior habían participado en su gestación y discusión; todo ello con el respaldo del Partido por la Democracia (PPD). La acción que inicialmente desplegó la CONADI se mantuvo cerca de las organizaciones más involucradas. La separación entre institución del Estado y organización fue desde ya un problema. En los primeros tiempos de la Subdirección Norte de la CONADI, quizá los dos o tres primeros años (1993-1995), resultaba bastante difícil separar la acción de la CONADI de la acción de las organizaciones cuyos principales dirigentes, líderes e intelectuales nutrieron las direcciones provinciales y la Subdirección Nacional. Se asumió, aparentemente, la opción de un potenciamiento de la acción de las organizaciones con base en una presencia e instalación estratégica en el organismo creado por el Estado; es la idea de una red articulada desde la institución, pasando por las organizaciones de origen, más los profesionales e intelectuales que se reclutaron, para desde allí trabajar una cobertura más amplia.

Una estrategia de este tipo pudo ampliar la acción y la influencia de las organizaciones étnico reivindicativas, pero también contenía problemas potenciales. Uno de ellos es el peligro de confusión entre acción colectiva y acción burocrática. Ella acarreó observaciones e intervención de las autoridades y los organismos contralores de la acción del Estado (además de dejar en el ambiente una percepción de corrupción). Otro, es la confusión de intereses políticos del movimiento e intereses partidarios. Esto se tradujo en la crítica y la presión des de los partidos políticos regionales por un alineamiento partidario menos ambiguo, más definido en favor de la Concertación. ${ }^{46}$ Un tercero, es el de la competencia por recursos desde las organizaciones de base y, en asociación con ello, la cuestión del reconocimiento. Esto se manifestó en forma de crítica y discenso respecto de las modalidades de asignación y distribución de recursos (acusaciones de favoritismo, nepotismo, etc., de las que también se hicieron eco autoridades, partidos y funcionarios de otras reparticiones públicas). Un cuarto, es la dependencia del paraguas institucional y los recursos provistos por la CONADI para cualquier tipo de

46 Las críticas tampoco faltaron desde la oposición, ya no por omisión sino que por la no separación entre acción del Estado y acción político partidaria. acción. Es dudoso, se podría pensar, que las organizaciones y redes se mantengan activas si falla el apoyo y los recursos financieros del Estado. No podría ser de otro modo: es una red de adeptos y partícipes, pero también de clientes. Otro más, aun, es el de la competencia por clientelas en los espacios comunales, donde las posibilidades de los grupos que compiten por el municipio y de los propios gobiernos comunales son muy amplias (lo cual se manifestó bajo la forma del descrédito, acusaciones de corrupción e ineficiencia, desconocimiento, etc.).

Diversas medidas se desarrollaron desde la situación creada durante los primeros años. Son acciones dirigidas a separar los ámbitos de competencia y acción de la CONADI y las organizaciones, a ampliar la cobertura de la acción, a la formación y extensión de clientelas y a crear y ejercer una cierta influencia política. Las llaves maestras de las tres primeras cuestiones fueron la elaboración de un Plan de Desarrollo Andino y la creación del Consejo Nacional Aymara (CNA) y, la de la última, el lanzarse a la competencia política en los planos en que ello era posible. Antes de desarrollar la dinámica generada a partir del Plan de Desarrollo y el Consejo Nacional, consignaremos algunos elementos informativos de la ación política, bajo el entendido de que este tema por si solo puede ser materia de un análisis mucho más extenso que escapa a los alcances de este trabajo.

La participación política se dio en varios niveles. Su resultado no es exitoso, pero tampoco fue del todo inocuo. Primero, la participación en el juego político de las comunas. En las elecciones municipales de 1996 se presentó con apoyo no oficial de la CONADI a numerosos candidatos a consejeros por las ocho comunas rurales con presencia aymara importante en la región de Tarapacá. Los resultados fueron un fracaso completo, salvo en aquellos casos en que se había logrado acuerdos con líderes y personalidades locales (como en Colchane, por ejemplo). Candidatos no locales, de extracción urbana, poco conocidos en las comunas, con un discurso intelectualizado y ribetes indianistas no convocaron mayor interés. Es el costo de no poderse desprender, todavía, de una relación muy cerrada con las organizaciones de origen ${ }^{47}$ Se intentó, tam-

47 Fundamentales para el proceso que condujo finalmente al acceso a la CONADI, pero bastante menos útiles como mediación en la relación entre CONADI, la elite que la controlaba y una base de población ampliada. 
bién sin mayor éxito, salvo en el caso de la comuna de Putre, lograr la designación de consejeros regionales afines que integraran el consejo asesor del Intendente Regional y que intervienen en la confirmación de las propuestas de asignación de fondos regionales. Los partidos de derecha, debido al fuerte peso electoral que mantienen en estas comunas rurales lograron la elección de la mayoría de los consejeros. Un mejor control, por encontrarse dentro de sus posibilidades de dominio, y por falta de competencia, tuvieron en la designación de un representante ante el Fondo Indígena y en el nombramiento del consejero aymara ante el Consejo $\mathrm{Na}$ cional de la CONADI. En estos casos lograron imponer, prácticamente sin contrapeso, miembros calificados de su propio grupo. No obstante, ya en un momento tardío como inicios de 2000 , la elección del consejero aymara en la CONADI de la administración de Ricardo Lagos evidenció una considerable pugna interna de una élite que, pese a diferencias, ${ }^{48}$ se había mantenido estratégicamente unida. Otro tanto ocurrió con la elección del consejero atacameño.

El Plan de Desarrollo Andino se consagró en el primer congreso aymara (para efectos ulteriores el primer congreso nacional aymara) celebrado en Arica en octubre de 1996. Se logró convocar a dirigentes y representantes de un buen número de organizaciones de base, urbanas y rurales. El interés del Plan no radica tanto en su contenido (en lo principal, una propuesta que responde a una demanda desarrollista con algunos elementos de demanda étnica), como en sus implicaciones. Una de ellas es que la Subdirección Nacional Norte y sus oficinas provinciales contraen un compromiso con un plan que aparece mandatado por una base amplia de organizaciones. De esta manera, la CONADI y el grupo que la controla logra una audiencia, una autoridad y una legitimidad que, como se dijo, se encontraba cuestionada. Sobre la base de lo anterior se posibilita una ampliación significativa en su cobertura de trabajo con organizaciones de base. Ciertamente, con ello también se busca la constitución de clientelas que pudieran servir como una base de apoyo fortalecido para el propio grupo en la CONADI y como soporte para un mejor desempeño electoral de la Concertación. Nuevamente, la competencia por clientelas por parte de otras agencias estatales y los

48 Competencia entre Arica- Parinacota e Iquique, competencia entre visiones de etnicidad y estilos de acción, etc. gobiernos comunales, además del desarrollo de lógicas oportunistas en las propias agrupaciones y organizaciones locales, hace bastante relativo el éxito alcanzado. ${ }^{49}$

La autoridad y la legitimidad que provee el Plan no sólo se relaciona con la acción hacia las organizaciones de base, sino que también hacia el Estado. El Plan es un instrumento político para la acción de la CONADI hacia el propio Estado. Por consistir en, o aparecer como demanda étnica sistematizada, este se constituye también en una referencia que no pueden obviar los demás órganos del Estado y los gobiernos comunales y viabiliza el mandato de coordinación de acciones hacia los indígenas que le impone la ley que crea su institucionalidad.

La creación del Consejo Aymara en el primer congreso se planteó ante la necesidad manifiesta de contar con un órgano originado en las bases indígenas, que al menos apareciera como autónomo de la CONADI y que tuviera por mandato "direccionar" (definir y encauzar) la acción de base y la demanda agregada en un nivel étnico. En el segundo congreso realizado en la ciudad de Iquique a finales de mayo de 1997, el Consejo se redefine como Consejo Nacional Aymara, pasando de ser un órgano regional surgido de las tensiones que arrastraba la CONADI en sus primeros años de desempeño, a una entidad de representación nacional. ${ }^{50}$

En su conformación se intenta incorporar representantes miembros de las organizaciones históricas del grupo que controla CONADI, afines a su línea de acción. Esto se logra en el primer Consejo, formado en 1996, pero en el segundo, elegido a inicios

49 Por lo demás, la CONADI moviliza relativamente pocos recursos en el norte del país; tampoco es exitosa en convocar adecuados niveles de coordinación entre los organismos del Estado y los municipios; el discurso indigenista de la CONADI no es hegemónico, de lo cual resulta que sus clientelas son inestables, movedizas, acomodaticias y oportunistas. De esta manera, no es raro que las organizaciones de base tengan caras organizativas múltiples en función de las ofertas del Estado. La exageración de esta situación se vive en las comunidades atacameñas.

50 Sus objetivos son: 1) Representar al Pueblo Aymara organizado a escala nacional constituyéndose como su vocero, ante el Estado y sus instituciones, ante la sociedad civil chilena y en el ámbito internacional; 2) promover las medidas tendientes a canalizar las demandas económicas, políticas y culturales del Pueblo Aymara, a fin de impulsar su desarrollo integral de acuerdo a su tradición cultural y realidad actual, para lo cual toma como instrumento el Plan Estratégico de Desarrollo Andino. 
del año 2000, esa posibilidad es más lejana. A partir de ello se abren nuevas condiciones para esta estructura de representación: una es la autonomía de la CONADI y, con ello, como una segunda cuestión, la diversificación de intereses que el Consejo puede expresar.

En su primera versión, la composición del Consejo Aymara integró a ocho consejeros, cuatro de Arica ${ }^{53}$ y cuatro de Iquique. ${ }^{52}$ En los hechos las representaciones se ajustaban a la necesidad de un equilibrio Arica Parinacota-Iquique, y a la colocación de consejeros favorables a la administración de la Subdirección o sus oficinas; es el caso, por ejemplo, de la situación de Iquique que consideraba a dos consejeros. En la segunda versión, el padrón se modificó, restando un consejero a Iquique y asignando ese cupo a la representación de aymaras fuera de la I Región de Tarapacá (Calama, Antofagasta y Región Metropolitana). A diferencia de los consejeros nacionales que se eligen según un padrón que, al menos durante la última elección, controla el Ministerio de Planificación (MIDEPLAN), los miembros del Consejo Nacional los eligen las organizaciones ${ }^{53}$ en los congresos nacionales aymaras.

Aun cuando en sus orígenes el Consejo aymara se planteó acciones hacia adentro, en los hechos actúa sólo o principalmente hacia fuera. Su denominación vernácula es la de un Consejo de Mallkus y Thallas (consejo de hombres y mujeres con responsabilidad y autoridad colectivas). Esta denominación, más allá de la simbolización étnica que lleva implicada (junto a la parafernalia de que se envisten en sus apariciones públicas), recuerda uno de los contenidos de que se rodeó su creación: constituir un cuerpo de autoridades que actúa en el plano moral, religioso y del derecho consuetudinario como una suerte de guardianes de la cultura. La inactividad en este plano se contraresta con el dinamismo desplegado en representar ante el Estado en los niveles provinciales, regional y nacional. Busca ser un interlocutor y representante autorizado en un nivel étnico agregado para la gestión de la demanda aymara. Intenta influir en el gobierno y en los niveles mencio-

51 Uno por cada comuna: Arica, General Lagos, Putre y Camarones.

52 Uno del altiplano, comuna de Colchane; uno precordillerano (Huara, Camiña, Pica y Pozo Almonte) y dos urbanos para Iquique, en concordancia, se decía, con la distribución de la población

53 De todo tipo, locales, sectoriales y étnicas de cada una de las jurisdicciones indicadas: siete comunas y resto del país nados las decisiones que directa o indirectamente inciden sobre lo andino.

Lo anterior lo han venido haciendo de manera autónoma, sin la mediación o de manera independiente de la CONADI, como asimismo sin la participación del Consejero Nacional, a quien para ciertos efectos han simplemente desconocido u obviado. Por lo demás, y con razón, el Consejero anterior y el actual son vistos como miembros relevantes de la red de poder e influencia que tejió el grupo que controla la Subdirección Norte de la CONADI. En un sentido, entonces, puede entenderse como una pugna de facciones de la élite aymara por el control de influencias y cuotas de poder. En efecto, puede considerarse a la mayoría de los miembros del Consejo Nacional como dirigentes y líderes de segunda y/o de segunda generación.

Además de lo ya dicho, interesa destacar algunas consecuencias importantes. La primera y más obvia es, por cierto, que la representación aymara se ha definitivamente diferenciado de la CONADI o, para ser más exactos, del grupo de élite que desde 1990 controla en el norte de Chile primero la CEPI y, desde 1993, la CONADI. Esta, a su vez, por la presión ejercida por otros nucleamientos de intereses andinos, se desprende de la compleja relación que por alrededor de una década mantuvieron con la población andina. En esta medida, la función de mediación y la red de mediación que desarrollaron está evolucionando rápidamente, desapareciendo la una, descomponiéndose la otra. En segundo lugar, la propia representación andina ha cambiado de sentido. No es ahora orquestada desde la élite, sino que mucho más democrática. La manipulación sigue dándose y no es un atributo exclusivo de la élite que analizamos, pero hay mucho mejores condiciones para una generación de representantes autónoma. En esa medida, la propia representación se ha diversificado más (más organizaciones representadas, mayor expresión de la diversidad de intereses). En tercer término, el Consejo Nacional ha buscado presionar a la propia CONADI en función de una composición de la institución más acorde con sus intereses y un estilo de acción consecuente con sus prioridades de demanda. Hasta cierto punto lo han conseguido. ${ }^{54}$ Asumen así una posición activa,

54 Por ejemplo, durante la primera mitad del 2000 incidieron en que el nuevo director de la Subdirección Norte fuera no sólo un aymara, cosa que dan por descontado, sino que además alguien de fuera del círculo del director saliente, o del ámbito de confirmación de éste. 
propositiva y de presión en el Estado ante todo aquello que les concierna (nombramiento de funcionarios incluido).

Surgen, no obstante, un par de problemas. De una parte, la derechización del Consejo Nacional a partir de la influencia que ha venido ejerciendo un consejero regional aymara que actúa como asesor del Consejo en Iquique y de miembros del Consejo que son afines con partidos de derecha, que lo pueden orientar hacia una cierta visión política y a poner en entredicho los nexos ya prolongados de intelectuales y líderes aymaras con la Concertación a través del Partido por la Democracia. Con ello, puede romperse un frágil lazo entre la coalición gobernante y la población aymara que, aunque en evolución, durante la última década es votante mayoritaria de los partidos de derecha. Derechización también en el sentido de reforzar una demanda desarrollista en desmedro de un perfilamiento étnico del desarrollo. Un segundo tema problemático surge así con el indigenismo que levanta el Consejo. Este no es indianista, sino más bien siguen una idea indigenista clásica. Sus miembros son especialmente sensibles a una demanda de desarrollo y también étnica, pero no integrada en una propuesta mayor de pueblo, que apunte en la dirección de la autonomía. En este sentido particular el Consejo sigue siendo una instancia de representación étnica unitaria, pero funcional a la política general del gobierno; por cierto, mientras la derecha política no gane mayores posiciones. La promesa de un movimiento social andino en el norte de Chile sigue esperando mejores tiempos. ${ }^{55}$

\section{Conclusiones}

Este trabajo presenta un análisis de la etnogénesis andina en el norte de Chile durante las dos décadas recién pasadas; trata centralmente entonces acerca de la constitución de aymaras, atacameños y collas como actores étnicos. Este proceso representa una novedad histórica ya que durante el último siglo la acción colectiva, defensiva o proactiva, de la que participaron esas poblaciones, se estructuró sólo según un formato de clase (aunque como mera pre-

55 Este análisis cubre el proceso étnico regional hasta, aproximadamente, mediados del año 2000. Con posterioridad se han producido diversos acontecimientos largos de detallar que, sin contradecir el análisis presentado, lo llevarían por nuevos derroteros. sencia andina en las luchas sindicales de los enclaves mineros del desierto), localista (como acción de las comunidades rurales frente al mercado o al Estado) y sectorial (como agregación de intereses que van más allá de la comunidad o lo atraviezan). Un antecedente importante de este cambio es la presión ejercida por el mercado para la enajenación de recursos productivos indígenas (aguas para la minería, principalmente) y la existencia de un gobierno autoritario cuya legislación. políticas y acción favorecían abiertamente al capital. Con el apoyo de ONGs y de manera autónoma, dirigentes de base y una intelectualidad andina incipiente crean organizaciones y desarrollan acciones de defensa de tales recursos; en torno a ellas se enarbolan ahora las banderas de la etnia aymara. Para que esto fuera posible debieron estar disponibles sensibilidades y discursos étnicos, así como sujetos que los incorporan y que plantearan su acción con arreglo a ellos.

Junto al inicio de una transición democrática inconclusa se desarrolla un pacto entre la Concertación (coalición política gobernante) y las organizaciones étnicas andinas (atacameños incluidos, que ahora se suman al proceso iniciado por los aymara). Aquel conduce al nacimiento de la CONADI (Corporación Nacional de Desarrollo Indígena), organismo que incluirá entre sus funcionarios a los dirigentes y iíderes más connotados. Estos se transforman en mediadores de doble faz (funcionarios públicos y representantes de organizaciones), hasta momentos recientes en los cuales progresivamente esas funciones opuestas se separan. El instrumento para ello es el Consejo Nacional Aymara. Desde entonces no hay movimiento propiamente, sino una base organizacional ampliada que genera demandas colectivas, algunas con contenidos étnicos, las cuales son canalizadas a través de los organismos estatales ad hoc. La dimensión de lo étnico en la relación del Estado con las poblaciones étnicas al parecer vino para quedarse (en la acción de organismos sectoriales y en los gobiernos locales, especialmente), pero la lógica de la acción y su dinámica se ha modificado. Se pasa de sujetos étnicos defensivos a otros que mantienen un estatuto de conveniencias mutuas con el Estado para beneficiarse de su acción desarrollista. 


\section{BIBLIOGRAFIA}

GONZALEZ, H. y GUERRERO, B., 1990. Desarrollo andino y cultura aymara en el norte de Chile, Taller de Estudios Aymaras (TEA) y Centro de Investigaciones de la Realidad del Norte (CREAR), Iquique, Chile.

FOERSTER, R. y VERGARA J. I., 2000. Etnia y nación en la lucha por el reconocimiento. Los mapuches en la sociedad chilena en Estudios Atacameños No 19: 11-42, San Pedro de Atacama.

GUNDERMANN, H., 1997. Etnicidad, identidad étnica y ciudadanía en los países andinos y el norte de Chile. Los términos de la discusión y algunas hipótesis de investigación. Estudios Atacameños 13: 9-26, San Pedro de Atacama.

-..-1 1998. Comunidad aymara, identidades colectivas y Estados nacionales en los albores del S.XX. En $A 90$ años de los sucesos de la Escuela Santa María de Iquique, DIBAM, LOM, CIDB, U. Arturo Prat, Iquique, Chile, pp. 153-181.

---- 1999. Comunidad indígena y ciudadanía: La experiencia aymara en el norte de Chile. Allpanchis 46: 91-130, Instituto de Pastoral Andina, Sicuani, Cuzco, Perú.
MELUCCI, A., 1996. Challenging Codes. Collective action in the information age. Cambridge University Press, Cambridge, United Kingdom.

--- 1998. "La acción colectiva como construcción social" en Estudios Sociológicos IX, N²6: 357-364, México.

PTI, 1989. "Partido de la Tierra y la Identidad. Declaración de principios" en Nütram, Año V No 1, pp. 2-3.

\section{Documentos}

Partido de la Tierra y la Identidad (PTI). Declaración de Principios, Santiago, marzo de 1989.

Segundo Acuerdo de los pueblos indígenas de Chile, Niagua 27 y 28 de enero de 1990

Estrategia de Desarrollo Aymara, Consejo Nacional del Pueblo Aymara (Mallku - T'alla), 1998. Iquique, Chile.

Informe de la comisión asesora en temas de desarrollo indígena, Mideplán, 1999, Santiago, Chile. 
